\title{
An analytical model of heat transfer and fluid dynamic performances of an unconventional NTR engine for manned interplanetary missions
}

\author{
Ivan Di Piazza \\ Dipartimento di Ingegneria Nucleare, Universitá degli studi di Palermo, Viale delle Scienze, Edificio 6, CAP 90128, Palermo, Italy
}

\section{A R T I C L E I N F O}

\section{Article history:}

Received 29 December 2008

Received in revised form 2 September 2009

Accepted 18 September 2009

\begin{abstract}
A B S T R A C T
An analytical model of fluid flow and heat transfer of a Nuclear Thermal Rocket (NTR) engine concept is presented. The engine is based on the direct conversion of the kinetic energy of the fission fragments (FFs) into the propellant enthalpy. The FFs can escape from an extremely thin layer of fissionable material: a sufficiently large surface coated with few micrometers of Americium $242 \mathrm{~m}$, confined by a neutron moderator-reflector, may become a critical reactor.

Three dimensional coupled CFD-Monte Carlo simulations have already been presented in Di Piazza and Mulas (2006). In this paper, an analytical integral 1-D model of fluid dynamics and heat transfer is built in order to foresee the performances on the basis of simple, physically founded correlations. The Peclet number has been identified as the main governing parameter of the system, and theoretically based correlations have been found for the thermodynamic efficiency of the engine and for the specific impulse. The correlations show a good agreement with numerical results presented in Di Piazza and Mulas (2006) from fully coupled 3D CFD-Monte Carlo calculations.
\end{abstract}

(c) 2009 Elsevier B.V. All rights reserved.

\section{Introduction}

Nuclear Thermal Rocket propulsion is considered the only technology that can lead to manned interplanetary missions (De Luca, 2003; Grey, 2004). This is due to the fact that thermal Nuclear propulsion can reach a specific impulse ${ }^{1}$ much higher than chemical propulsion due to the higher enthalpy level of the propellant in the stagnation chamber; this topic is fully discussed in Di Piazza and Mulas (2006). The higher possible value of the specific impulse is crucial for interplanetary missions, as it is directly related to the rocket mass by the standard rocket equation:

$M_{p}=M\left(e^{\Delta V / I_{s p}}-1\right)$

where $M_{p}$ is the mass of propellant needed for the mission, $M$ is the total burnout mass of spaceship and $\Delta V$ is the velocity change required for the mission. For a given mission, a higher $I_{s p}$ implies a lower mass of propellant $M_{p}$ to be carried. The large amount of propellant to be carried is considered the main limiting factor for manned interplanetary missions.

A review of the evolution of the Nuclear rocket propulsion is presented in Di Piazza and Mulas (2006), see Borowski et al. (1989),

\footnotetext{
E-mail address: ivandipiazza@yahoo.it.

1 The specific impulse represents the thrust force exerted by the unit propellant mass flow rate and, in S.I. units, has the dimension of a velocity $[\mathrm{m} / \mathrm{s}]$ and it is almost equal to the exhaust velocity of the propellant at the nozzle exit section; it can be also expressed in seconds dividing by the gravity acceleration $g$.
}

Frisbee (2003), Grey (2004), Howe (2000); a new nuclear rocket concept has been recently introduced by Rubbia (2000) and it is based on an idea also proposed by Chapline (1988), Ronen and Shwageraus (2000) and Kammash et al. (1993), among others in the past two decades. Fission fragments escaping an ultra thin layer of few micrometers of Americium 242m can be used to heat up a hydrogen flow. Heavy ionising fragments produced by neutron induced nuclear fissions carry the major part of the corresponding fission energy in the form of kinetic energy (about $168 \mathrm{MeV}$ out of approximately $191 \mathrm{MeV}$ per fission event). FFs convert all of their kinetic energy into internal energy of the propellant gas, slowing down via electromagnetic interaction, provided that a sufficient effective thickness ${ }^{2}$ of propellant is present (about $500 \mu \mathrm{g} / \mathrm{cm}^{2}$ (Rubbia, 2000)). By this unconventional (direct) heat transfer mechanism, the enthalpy of the gas in the stagnation chamber can reach values of about $200-300 \mathrm{MJ} / \mathrm{kg}$, at low pressures of few bars, corresponding to temperatures of about $4000-4800 \mathrm{~K}$ and to a specific impulse $I_{s p}$ of the order of $15,000-20,000 \mathrm{~m} / \mathrm{s}$.

Due to the direct energy conversion (unconventional heat transfer) this new concept of NTR shares the same "cold" structure-"hot" propellant configuration of the gas-core nuclear rocket concept, thus outperforming the conventional NTR concept: the hydrogen

\footnotetext{
2 the effective thickness is defined as the mass of propellant per unit area of Americium $242 \mathrm{~m}$, and it can be seen as the mass of propellant available to slow down fission fragments.
} 


\begin{tabular}{|c|c|}
\hline \multicolumn{2}{|c|}{ Nomenclature } \\
\hline$c_{p}$ & specific heat of the gas $[\mathrm{J} / \mathrm{kg} / \mathrm{K}]$ \\
\hline$d Q_{\text {conv }}$ & $\begin{array}{l}\text { power extracted by convection in the volume } d V \\
\text { [W] }\end{array}$ \\
\hline$d Q_{\text {wall }}$ & power lost by conduction in the volume $d V$ [W] \\
\hline$d Q_{g e n}$ & power deposited in the volume $d V$ [W] \\
\hline$d V$ & $\begin{array}{l}\text { elementary volume of the module used to write bal- } \\
\text { ance equations }\left[\mathrm{m}^{3}\right]\end{array}$ \\
\hline$D$ & module diameter $[\mathrm{m}]$ \\
\hline$h$ & average propellant stagnation enthalpy [J/kg] \\
\hline$h_{\text {wall }}$ & $\begin{array}{l}\text { average propellant stagnation enthalpy before } \\
\text { entering heating chamber }[\mathrm{J} / \mathrm{kg}]\end{array}$ \\
\hline$I_{s p}$ & specific impulse $[\mathrm{m} / \mathrm{s}]$ \\
\hline$I_{S p R E D}$ & nondimensional reduced specific impulse \\
\hline$I_{S P S C A L E}$ & specific impulse scale $[\mathrm{m} / \mathrm{s}]$ \\
\hline$L$ & module length [m] \\
\hline$M$ & total burnout (final) mass of spaceship [kg] \\
\hline$M_{p}$ & mass of propellant needed for the mission $[\mathrm{kg}]$ \\
\hline$\dot{m}_{p}$ & engine mass flow rate $[\mathrm{kg} / \mathrm{s}]$ \\
\hline$\dot{m}$ & single module mass flow rate $[\mathrm{kg} / \mathrm{s}]$ \\
\hline$\dot{m}_{\text {wall }}$ & mass flow rate per unit wall area $[\mathrm{kg} / \mathrm{s}]$ \\
\hline$N$ & number of modules \\
\hline $\mathrm{Nu}$ & Nusselt number for conductive losses \\
\hline$P$ & propellant stagnation pressure [Pa] \\
\hline $\mathrm{Pe}$ & Peclet number \\
\hline $\operatorname{Pr}$ & Prandtl number \\
\hline$\dot{q}_{F F}$ & rate of fuel consumption $\left[\mathrm{W} / \mathrm{m}^{2}\right]$ \\
\hline$\dot{q}_{\text {wall }}^{\prime \prime}$ & wall heat flux $\left[\mathrm{W} / \mathrm{m}^{2}\right]$ \\
\hline$\dot{Q}_{\text {cond }}$ & power lost by heat conduction [W] \\
\hline$\dot{Q}_{F}$ & gross nuclear fuel power [W] \\
\hline$\dot{Q}_{\text {FFlost }}$ & nuclear fuel power entrapped in the fuel layer [W] \\
\hline$\dot{Q}_{h}$ & heating power available for generating thrust [W] \\
\hline$\dot{Q}_{\text {nozzle }}$ & power lost in the nozzle expansion [W] \\
\hline$\dot{Q}_{p}$ & propulsion power of the engine $[\mathrm{W}]$ \\
\hline $\operatorname{Re}$ & $\begin{array}{l}\text { Reynolds number based on the specific mass flow } \\
\text { rate }\end{array}$ \\
\hline $\mathrm{Re}_{\text {mod }}$ & proper module Reynolds number \\
\hline$S$ & surface of the heating tube $[\mathrm{m}]$ \\
\hline$T$ & average propellant stagnation temperature $[\mathrm{K}]$ \\
\hline$T_{\text {wall }}$ & wall temperature [K] \\
\hline$V_{\text {exit }}$ & propellant velocity at the nozzle exit $[\mathrm{m} / \mathrm{s}]$ \\
\hline$\delta f$ & fuel thickness [m] \\
\hline$\Delta T$ & average temperature drop in a module $[\mathrm{K}]$ \\
\hline$\Delta T_{\max }$ & maximum temperature drop in a module $[\mathrm{K}]$ \\
\hline$\Delta V$ & velocity change $[\mathrm{m} / \mathrm{s}]$ \\
\hline$\Delta h$ & hydrogen enthalpy gain in a module $[\mathrm{J} / \mathrm{kg}]$ \\
\hline \multicolumn{2}{|c|}{ Greek letters } \\
\hline$\alpha$ & themal diffusion of the gas $\left[\mathrm{m}^{2} / \mathrm{s}\right]$ \\
\hline$\eta_{\text {capture }}$ & FFs capture efficiency \\
\hline$\eta_{\text {layer }}$ & Americium layer efficiency \\
\hline$\eta_{F F}$ & fission fragments efficiency \\
\hline$\eta_{h}$ & heating efficiency \\
\hline$\eta_{N}$ & nozzle efficiency \\
\hline$\eta$ & overall efficiency \\
\hline$v$ & kinematic viscosity of the gas $\left[\mathrm{m}^{2} / \mathrm{s}\right]$ \\
\hline
\end{tabular}

flow may enter the stagnation chamber at low temperature (say in the range $1500-2000 \mathrm{~K}$ ) and can heat up, in principle without limits, flowing towards the nozzle.

The aim of this paper is to present an analytical study of heat transfer and fluid dynamic performances of the engine alone. The- oretical correlations are therefore derived and compared with numerical fully 3D results presented in Di Piazza and Mulas (2006). The theoretical analysis shows that the dominant parameter of the system is the Peclet number, and the performances are therefore linked to this nondimensional parameter.

The scientific feasibility of the FF unconventional NTR concept has been demonstrated in Rubbia (2000), while reference Di Piazza and Mulas (2006) presents an integrated software system for simulating the fluid dynamic and thermal behaviour of the system.

In reference Di Piazza and Mulas (2006), the analysis has been performed on a single heating tube (module) with 3D axisymmetric simulations using a hybrid integrated software system. The system is made of a CFD code (Mulas et al., 2002) coupled to a neutronic code and to a Monte Carlo code. The neutronic code, for a chosen fissile material and fuel layer thickness, computes a database containing the power density (in $\mathrm{W} / \mathrm{m}^{2}$ ) per unit fuel area, the percentage of FFs escaping the fuel layer and the kinetic energy distribution of the FFs. The outputs of the neutronic code are then used by the Monte Carlo code to model the fission fragments emission from the fuel layer and the direct energy conversion into propellant enthalpy. The CFD code computes the flow inside the engine.

\section{Engine description}

\subsection{General layout}

The conceptual design is based on a number $N$ of cylindrical modules (heating tubes) immersed in a bath of boiling lithium at a temperature $T_{L i} \approx 2000 \mathrm{~K}$. Fig. 1 shows a schematic representation of the engine. The tubes are internally coated with an extra thin layer of ${ }^{242 \mathrm{~m}} \mathrm{Am}$ : due to the high fission cross-section of ${ }^{242 \mathrm{~m}} \mathrm{Am}$ (about 5000 barns against 583 barns of ${ }^{235} \mathrm{U}$ for thermal neutron at

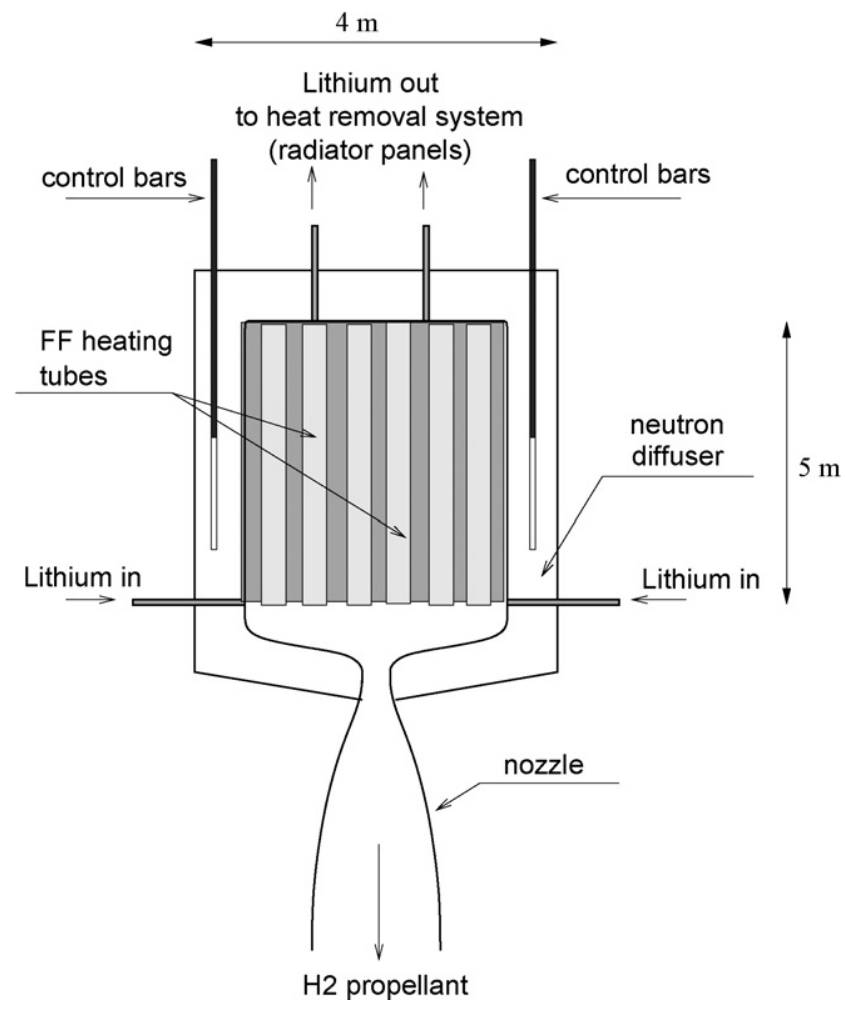

Fig. 1. Schematic representation of the FF heated propulsion engine. The configuration is a critical reactor cooled by boiling lithium and confined by a neutron moderator-reflector. The heating chamber is made of a number of cylindrical tubes immersed in a lithium bath. The heating tubes are $5 \mathrm{~m}$ long, while the external diameter of the engine is $4 \mathrm{~m}$. 


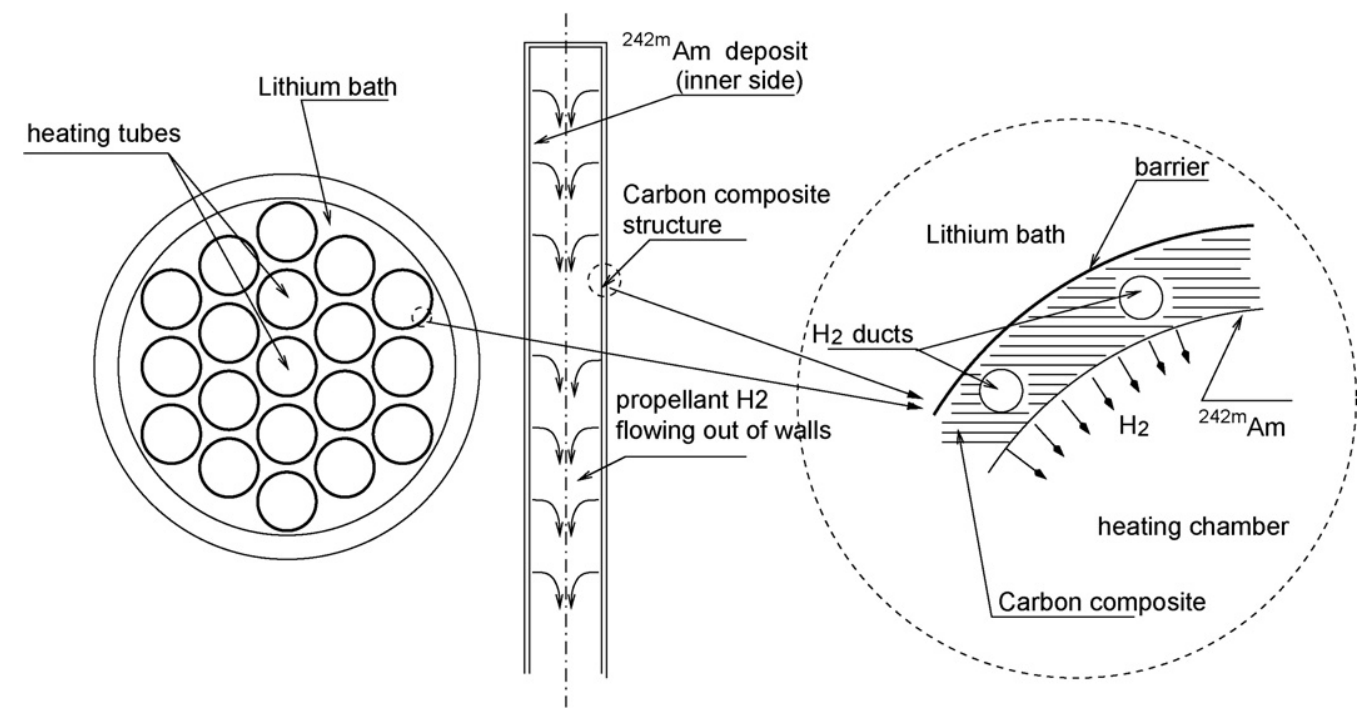

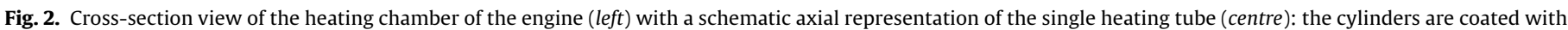

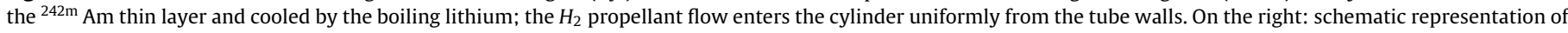
the permeable tube wall, based on the use of carbon composite fibers and matrices.

$0.025 \mathrm{eV}$ ), such a configuration represents a critical thermal reactor confined by a neutron reflector-moderator made of carbon (graphite) or beryllium (Be or BeO), and a sort of n-hohlraum can be created, assuring a multiplication factor $k_{\text {eff }}$ sufficiently high to give allowance of a reasonable burn-up, even for a fissile layer of few micrometers of thickness. Hydrogen enters the tubes uniformly, permeating the solid walls, and heats up as it flows out of the heating tubes by stopping the FFs.

Fig. 2 shows the so called Catiuscia (Benetti et al., 2005) arrangement of $N$ heating tubes of diameter $D$. The tubes structure is made out of carbon composite fibers and matrices, a material which is porous and permeable to hydrogen. A slab with thickness of the order of $1-2 \mathrm{~cm}$ with a limited number of narrow, hollow channels in which hydrogen can be introduced under pressure can provide the necessary mass flow rate of propellant $\dot{m}_{p}$. The thermo and fluid dynamic conditions of the propellant flowing inside the heat- ing tubes depend on the volumetric heat addition coming from the FFs: the energy deposit, proportional to the local propellant density, heats up the propellant and so changes its temperature and density, modifying in turn the FFs energy deposit itself. The final state of the propellant flow exiting the tubes, as well as the thermodynamic state in terms of temperature and pressure, comes from the equilibrium among many different effects: the conservation equations of mass, momentum and energy including a model for the FFs heating (Rubbia, 2000) and the $\mathrm{H}_{2}$ dissociation. Once the fluid has been heated, it flows into the stagnation chamber, and then it is accelerated through a conventional convergent-divergent nozzle, where the internal energy is converted into kinetic energy generating thrust. The portion of nuclear fission energy which is not converted into propellant enthalpy must be evacuated by the liquid lithium that flows out of the core region through small tubes to the radiating panels.

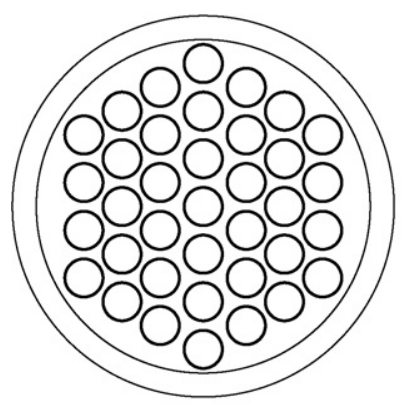

$N=37 D=0.4 m$

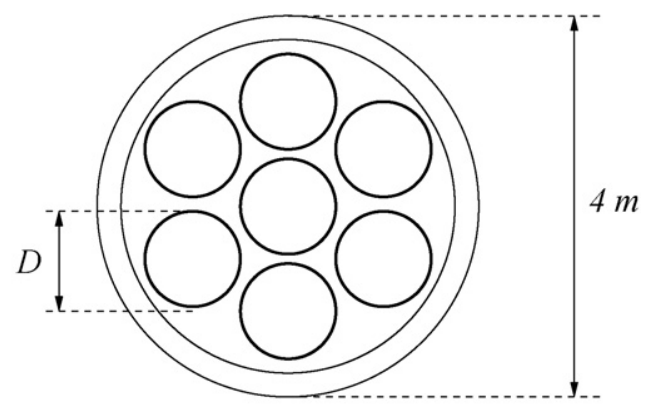

$N=7 D=1 m$

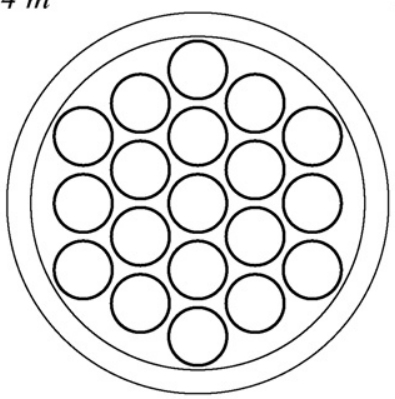

$N=19 D=0.6 \mathrm{~m}$

Fig. 3. Three possible Catiuscia configurations with an external diameter of $4 \mathrm{~m} . N=37,19$ or 7 respectively for modules of diameter $D=0.4$, 0.6 or $1.0 \mathrm{~m}$. 
The number of heating tubes (modules) which can be assembled together is limited by the size of the engine, which in turn is limited by the size of the cargo bay of the next generation Space Shuttle, for the engine has to be assembled in the International Space Station and can only be ignited in space. The dimension of the actual Space Shuttle cargo Bay are about $4.5 \mathrm{~m}$ in diameter and $18 \mathrm{~m}$ long. With the constraint of $D=4 \mathrm{~m}$, three multiple-module arrangements are shown in Fig. 3. The three configurations would fit 37, 19 and 7 modules, respectively, with diameter $0.4,0.6$ and $1 \mathrm{~m}$. A perspective view of the assembled Catiuscia engine is shown in Fig. 4, where the length of the modules has been fixed to $5 \mathrm{~m}$.

\subsection{Efficiency definition}

The number of fissions occurring in the engine per unit time and per unit surface of the fuel layer determines the rate at which energy is transferred to the propellant, or the rate of fuel consumption, $\dot{q}_{F F}$. The overall rocket engine efficiency $\eta$ is defined as the ratio between the propulsion power $\dot{Q}_{p}$ and the gross nuclear power available from the fission process $\dot{Q}_{F}$ :

$\eta=\frac{\dot{Q}_{p}}{\dot{Q}_{F}}=\frac{\dot{m}_{p} V_{e x i t}^{2} / 2}{N \pi D L \dot{q}_{F F}}$

where $V_{\text {exit }}$ is the propellant velocity at the nozzle exit, $S=\pi D L$ is the available emission surface of a single heating tube, $N$ is the number of heating tubes and $\dot{m}_{p}$ is the hydrogen total mass flow rate of the engine. The propellant leaves the Americium coated heating tube wall with an enthalpy level $h_{\text {wall }}$; it gains energy and goes to an average enthalpy $h$. If $\Delta h=h-h_{\text {wall }}$ represents the enthalpy increase due to the heating process, with respect to a initial (wall) enthalpy level $h_{\text {wall }}$, then the propulsion power can be computed as the heating stagnation power $\dot{Q}_{h}=\dot{m}_{p} \Delta h$ minus the nozzle losses:

$\dot{Q}_{p}=\dot{Q}_{h}-\dot{Q}_{\text {nozzle }}=\eta_{N} \dot{Q}_{h}$

$\dot{Q}_{\text {nozzle }}$ represents the losses associated with the expansion process in the convergent-divergent supersonic nozzle; these are due to

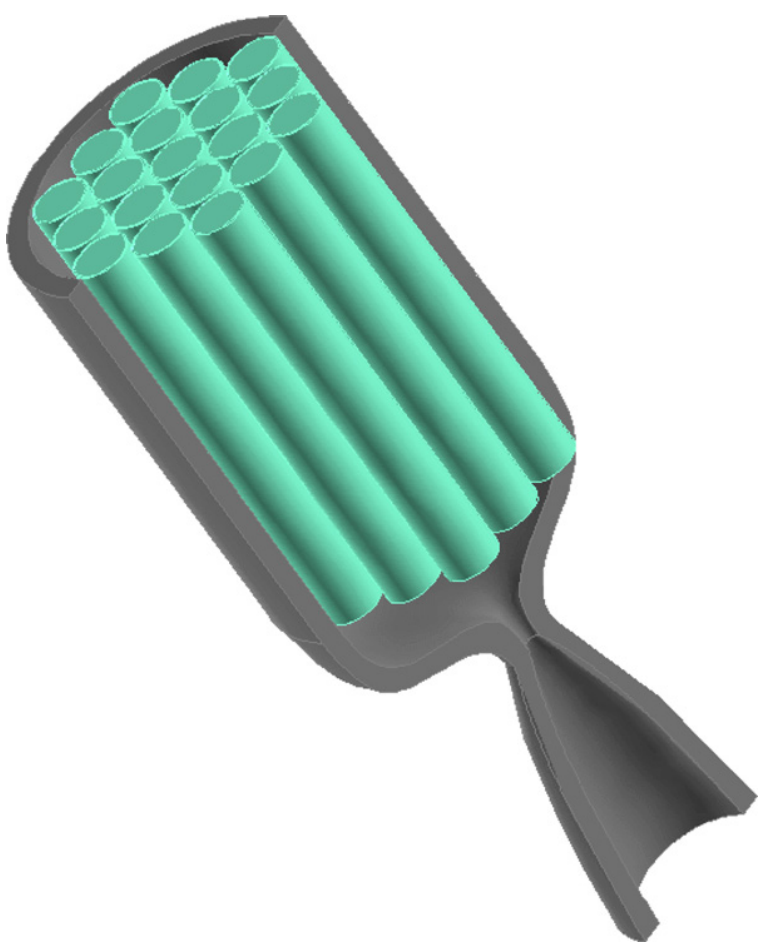

Fig. 4. Perspective view of the assembled Catiuscia engine. All modules discharge in the same nozzle. the missed chemical recombination into molecular hydrogen, and eventually to the frictional and heat flow losses through the nozzle walls. In Eq. (3) the nozzle efficiency $\eta_{N}<1$ has been introduced. The gross fission fragment power $\dot{Q}_{F}$ is converted into heating stagnation power $\dot{Q}_{h}$ with several losses. This power budget can be expressed as:

$\dot{Q}_{h}=\dot{Q}_{F}-\dot{Q}_{F F_{-} l o s t}-\dot{Q}_{\text {cond }}$

where $\dot{Q}_{F F \_l o s t}$ represents the power associated with the fission fragments that remain entrapped in the fuel layer or in the tube structure or escape from the system, and, hence, never becomes available for heating the propellant. The third term on the right hand side, $\dot{Q}_{\text {cond }}$, represents the power loss due to heat conduction through the tubes walls. The Reynolds number associated with the flow inside the heating tubes may, in fact, be low enough so that heat conduction losses can be important. The two sources of power which are lost return to the tube structure and need be evacuated by the lithium coolant, through the radiative panels. By introducing two more efficiencies $\eta_{F F}$, which takes into account the FF losses, and $\eta_{h}$ for the heat transfer losses, then the heating power $\dot{Q}_{h}$, representing the power which remains available for conversion into propulsive power, is given by:

$\dot{Q}_{h}=\left(\eta_{F F} \eta_{h}\right) \dot{Q}_{F}$

Alternatively we can introduce $\eta_{F F}=\eta_{\text {layer }} \eta_{\text {capture }}$,

$\dot{Q}_{h}=\left(\eta_{\text {layer }} \eta_{\text {capture }} \eta_{h}\right) \dot{Q}_{F}$

where the FF losses have been split into two contributions: one, $\eta_{\text {layer }}$, due to the FFs which leave part or all of their power inside the fuel layer (FFs that cannot escape from the thin layer, for instance), and the second one, $\eta_{\text {capture }}$ due to insufficient gas effective thickness present inside the tube to stop all available FFs escaping from the fuel layer. By combining all relations, an expression for the overall engine efficiency $\eta$ is given by:

$\dot{Q}_{p}=\left(\eta_{\text {layer }} \eta_{\text {capture }} \eta_{h} \eta_{N}\right) \dot{Q}_{F}=\eta \dot{Q}_{F}$

The layer efficiency, $\eta_{\text {layer }}$, depends on the fuel thickness (the thinner the layer, the higher $\eta_{\text {layer }}$ ), on the fuel chemical form (metallic rather than carbide, for instance) as well as on neutronic issues; it is always less than 0.5 for symmetry reasons (half of the FFs would tend to escape from the wrong side, converting their energy into heat of the wall structure). In this work, a layer of metallic ${ }^{242 \mathrm{~m}} \mathrm{Am}$ of thickness $\delta_{f}=3 \times 10^{-6} \mathrm{~m}$ and density $\rho_{F}=13,670 \mathrm{~kg} / \mathrm{m}^{3}$ has been assumed. Neutronic simulations based on these figures provides a value layer efficiency of $\eta_{\text {layer }}=$ 0.31 (Rubbia, 2000), which means that 70\% of the overall power $\dot{Q}_{F}$ is instantaneously converted into heat.

The second efficiency $\eta_{\text {capture }}$ measures the amount of power extracted by the FFs during the interaction with the flowing propellant; this value depends on the gas effective thickness, defined as the mass of the gas in a heating module per unit module area $\rho D / 4$. This quantity depends on the density of the gas which increases with the thermodynamic pressure. Its actual value depends on the design choices in terms of operating pressure and nozzle throat area: the higher the pressure, the higher $\eta_{\text {capture }}$ (eventually approaching unity), but this corresponds to a tighter throat area and higher nozzle losses (heat transfer losses in the nozzle). Values obtained in the simulations carried out, range between 0.75 and 0.95 .

The heating efficiency $\eta_{h}$ measures the power loss due to the heat conduction through the tube walls. The heating efficiency is the crucial efficiency, for it can vary widely between 0 and 1 , depending on the operating conditions. High mass flow rates (high Reynolds numbers) will yield high $\eta_{h}$, but low propellant specific enthalpy and specific impulse, and vice versa. 
Finally $\eta_{N}$ is the nozzle efficiency. The value of $\eta_{N} \approx 0.60$ represents a good approximation if the accelerating flow inside the nozzle remains in chemical equilibrium conditions, and if negligible viscous losses and wall heat transfer losses through the nozzle walls occur. This is attained if the nozzle dimensions are not too small; in fact a high Reynolds number inside the convergent-divergent nozzle yields negligible heat transfer losses through the nozzle walls. The design pressure in the stagnation modules appears as one of the critical design choices for the whole system. The lower possible pressure appears to be the optimal choice, provided that $\eta_{\text {capture }}$ remains at an acceptable level.

Considering the values discussed above, the overall engine efficiency is given by $\eta<0.16$. The power losses associated with $\left(\eta_{\text {layer }} \eta_{\text {capture }} \eta_{h}\right)$ represent the heat that must be removed by cooling the external walls by the liquid lithium loop, and by the radiative panels. If $\eta_{h} \approx 1$ such a power loss amounts to $74 \%$ (minimum) of the gross nuclear power.

The remaining $10 \%$ of the initial power is associated with the nozzle efficiency and mostly represents the power stored in the chemical bonds of atomic hydrogen (missed recombination of molecular hydrogen in chemical equilibrium conditions). This portion is directly lost towards deep space. The lower $\eta_{h}$, with respect to unity, the higher the power percentage that must be evacuated through the liquid lithium loop. The lithium loop should be designed to remove the total power produced with the reactor operating at $100 \%$ power to accommodate the case of zero propellant mass flow.

In this paper, the attention is focused on the heating efficiency $\eta_{h}$, which exhibits the largest variations, and thus is the crucial parameter to estimate. A simplified 1-D axial integral theory based on an energy balance is presented, and a theoretical correlation is derived for $\eta_{h}$ and $I_{s p}$.

\section{The analytical model of a single module}

\subsection{Nondimensional analysis}

If attention is focussed on the thermo-fluid dynamic performances of the single module only, i.e. on the heating efficiency $\eta_{h}$, the physical quantities describing the system can be identified as: the effective energy flux $\left(\eta_{F F} q_{F F}\right)$, the module diameter $D$, the module length $L$, the specific mass flow rate per unit wall area $\dot{m}_{\text {wall }}$, the kinematic viscosity $v$, the thermal diffusivity $\alpha$, the specific heat $c_{p}$ and the average enthalpy gain $\Delta h$. The Buckingham's theorem prescribes that the number of nondimensional parameters fully describing the system behaviour is given by the difference between the number of physical quantities involved (8) and the number of basic units used $(4, \mathrm{~m}, \mathrm{~kg}, \mathrm{~s}, \mathrm{~K})$; therefore 4 nondimensional parameters provides a full description of the system. These parameters are naturally selected as: the heating efficiency $\eta_{h}$, a module aspect ratio $L / D$, a Reynolds number Re (more precisely defined later), and the Prandtl number $\operatorname{Pr}=v / \alpha$. A functional relation exist between one parameter and the others; for example:

$\eta_{h}=f\left(\operatorname{Re}, \operatorname{Pr}, \frac{L}{D}\right)$

Developing the simplified theory these nondimensional groups would appear, in some sense, naturally, and the most appropriate definition of the Reynolds number will emerge from the formulation. The final aim of the theory is to find an analytical expression for the efficiency given in Eq. (8).

\subsection{The integral analytical model}

The model is based on the mass and energy balance of the single module schematically shown in Fig. 5.

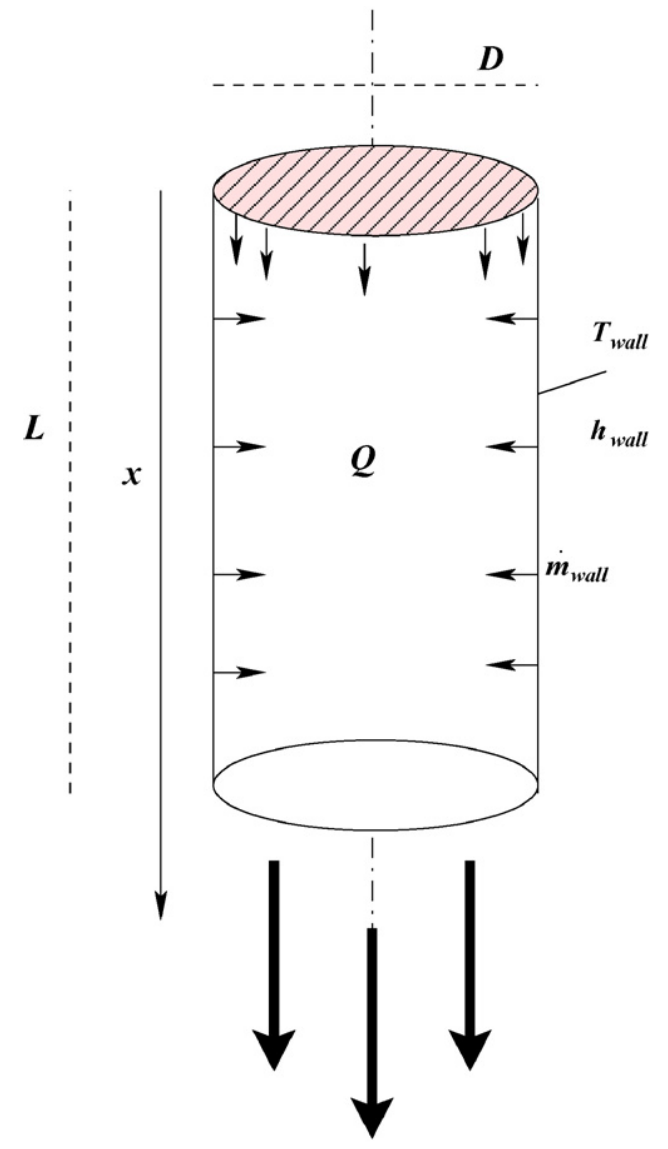

Fig. 5. Schematic representation of a single heating module.

The walls are kept at a constant temperature $T_{\text {wall }}$, and thus the gas is pre-heated while permeating the solid walls, and it will have the inlet enthalpy $h_{\text {wall }}$. Internal heat generation is provided by the fission fragments stopping in the chamber. This process can be approximately described by a constant power per unit volume $\dot{Q}\left[\mathrm{~W} / \mathrm{m}^{2}\right]$. The mass flow rate is provided from the solid wall at a constant specific rate per unit area $\dot{m}_{\text {wall }}\left[\mathrm{kg} / \mathrm{m}^{2} \mathrm{~s}\right]$. The module has a diameter $D[\mathrm{~m}]$ and a length $L[\mathrm{~m}]$. If an $x$ coordinate system is defined along the module length (see Fig. 5), the balances can be made with reference to an infinitesimal portion $d x$ of the module of volume $d V=\pi\left(D^{2} / 4\right) d x$. If $\dot{m}(x)[\mathrm{kg} / \mathrm{s}]$ is the mass flow rate of the gas at the generic section located at $x$, the mass balance prescribes:

$d \dot{m}=\dot{m}_{\text {wall }} \pi D d x$

The module mass flow rate at the section $x$ is then given by integration:

$\dot{m}(x)=\dot{m}(0)+\int_{0}^{x} \dot{m}_{\text {wall }} \pi D d x=\dot{m}_{\text {wall }} \pi \frac{D^{2}}{4}+\dot{m}_{\text {wall }} \pi D x$

The mass flow rate at the exit section located at $L$, can now be easily computed:

$\dot{m}(L)=\dot{m}_{\text {wall }} \pi \frac{D^{2}}{4}+\dot{m}_{\text {wall }} \pi D L$

The energy balance can be written in the following way:

$d Q_{g e n}=d Q_{\text {conv }}+d Q_{\text {wall }}$

where $d Q_{g e n}$ is the power deposited in the volume $d V$, while $d Q_{\text {conv }}$ and $d Q_{\text {wall }}$ represents the power extracted by convection and the power loss for heat conduction at the walls. Theoretical considerations (Rubbia, 2000) indicate that below 7000-8000 K, radiation 
losses become negligible, and therefore have not been considered in this analysis. The three pieces of Eq. (12) can now be estimated separately. The power generation in the volume $d V$ is simply:

$d Q_{g e n}=\dot{Q} \pi \frac{D^{2}}{4} d x$

If $h(x)$ is the average gas enthalpy at section $x$, the power extracted by convection in the volume $d V$ can be computed as:

$$
\begin{aligned}
d Q_{\text {conv }} & =\dot{m}(x+d x)\left(h(x+d x)-h_{\text {wall }}\right)-\dot{m}(x)\left(h(x)-h_{\text {wall }}\right) \\
& =(\dot{m}(x)+d \dot{m})\left(h(x)-h_{\text {wall }}+d h\right)-\dot{m}(x)\left(h(x)-h_{\text {wall }}\right) \\
& =\dot{m}(x) d h+\left(h(x)-h_{\text {wall }}\right) d \dot{m}
\end{aligned}
$$

neglecting the highest order infinitesimal quantities. Introducing the new variable $\Delta h(x)=h(x)-h_{\text {wall }}$, and using the continuity Eqs. (9) and (10), $d Q_{\text {conv }}$ has the final expression:

$d Q_{\text {conv }}=\left(\dot{m}_{\text {wall }} \pi \frac{D^{2}}{4}+\dot{m}_{\text {wall }} \pi D x\right) d(\Delta h)+\Delta h(x) \dot{m}_{\text {wall }} \pi D d x$

It can be shown that in realistic configurations, documented in $\mathrm{Di}$ Piazza and Mulas (2006), turbulence does not play a relevant role inside the tubes because the Reynolds number at the module exit section is at most 1000 for the largest values of module diameter and mass flow rate. Therefore, in this laminar flow condition, wall radial heat transfer mechanisms can be decoupled, i.e. the Nusselt number is independent from the Reynolds number. The effect of convection is simply to carry energy along the axial direction, and thus to produce different enthalpy (and temperature) levels according to the mass flow rate; higher mass flow rates lead to lower gas enthalpy levels.

To evaluate the wall heat flux, the pure conductive temperature distribution can be used. For a cylinder with constant heat generation per unit volume and constant wall temperature, the temperature distribution is parabolic:

$T(r)=T_{\text {wall }}+\Delta T_{\max }\left(1-\frac{4 r^{2}}{D^{2}}\right)$

where $\Delta T_{\max }$ is the maximum temperature drop between the axis and the wall. The wall heat flux can be computed as:

$q_{\text {wall }}^{\prime \prime}=-\left.k \frac{\partial T}{\partial r}\right|_{\text {wall }}=\frac{4 k \Delta T_{\max }}{D}$

With the parabolic distribution in Eq. (16), the ratio between the average temperature drop $\Delta T$ and the maximum temperature drop $\Delta T_{\max }$ is:

$\Delta T=\frac{3}{4} \Delta T_{\max }$

and thus the wall heat flux $q_{\text {wall }}^{\prime \prime}$ can be expressed with respect to the average drop as:

$q_{\text {wall }}^{\prime \prime}=\frac{16}{3} \frac{k \Delta T}{D}$

Defining a Nusselt number on the wall heat flux, as

$\mathrm{Nu}=\frac{q_{\text {wall }}^{\prime \prime}}{k \Delta T / D}$

a constant value of $N u=16 / 3$ is obtained in this case.

The total power exiting from the side walls in the volume $d V$ can the be written as:

$d Q_{\text {wall }}=q_{\text {wall }}^{\prime \prime} \pi D d x=N u \pi k \Delta T d x$
If this is expressed with respect to the average enthalpy drop $\Delta h$, the heat lost by conduction in a module becomes:

$d Q_{\text {wall }}=\mathrm{Nu} \pi \frac{k}{c_{p}} \Delta h d x$

Now the differential power balance equation in the module can be written from Eqs. (13), (15) and (22):

$\dot{Q} \pi \frac{D^{2}}{4} d x=\dot{m}(x) \frac{d(\Delta h)}{d x}+\left(\dot{m}_{\text {wall }} \pi D+\operatorname{Nu} \pi \frac{k}{c_{p}}\right) \Delta h(x)$

Eq. (23) is a total differential equation with variable coefficients, and the exact integration would lead to the function $\Delta h(x)$. In the limit, we can realistically approximate $x>>D$, i.e. $L>>D$; in this case thermal equilibrium is reached and only the particular solution survives. This concept has been confirmed by 3D numerical simulation, as shown in Fig. 6, where the axial distribution reaches an asymptotic behaviour close to the module exit section.

Under this hypothesis, the solution of Eq. (23) for $x>>D$ does not depend on $x$, i.e. it is a constant with respect to $x$. This solution can easily determined as:

$\Delta h=\frac{\dot{Q}\left(\pi D^{2} / 4\right)}{\dot{m}_{\text {wall }} \pi D+\mathrm{Nu} \pi\left(k / c_{p}\right)}$

From this solution, introducing the expression for the mass flow rate previously derived (Eq. (10)), with trivial passages, a clear analytical solution for the heating efficiency can be derived from the efficiency definition:

$\eta_{h}=\frac{\mathrm{Pe}}{\mathrm{Pe}+\mathrm{Nu}}$

where the Peclet number has been introduced as the product of Reynolds and Prandtl numbers:

$\mathrm{Pe}=\operatorname{Re}<\mathrm{CE}: \mathrm{HSP}$ SP $=" 0.25 " />\operatorname{Pr}=\frac{\dot{m}_{\text {wall }} D c_{p}}{k}$

The Prandtl number is, as usual, $\operatorname{Pr}=\mu c_{p} / k$, while a special Reynolds number is properly based on the specific wall inlet mass flow rate $\dot{m}_{\text {wall }}, \operatorname{Re}=\dot{m}_{\text {wall }} D / \mu$. This Reynolds number is related to the module outlet section Reynolds number $\mathrm{Re}_{\mathrm{mod}}$, through the relationship $\operatorname{Re}_{\text {mod }}=4 \operatorname{Re} L / D$. It should be noticed that the analytical expression for the heating efficiency in Eq. (25) does not depend on the module aspect ratio $L / D$ for a module length $L$ much larger than module diameter $D$. In the limit, we can realistically approximate $L>>D$.

In a similar way, from Eq. (24), it is possible to derive an analytical expression of the rocket performance in terms of nondimensional specific impulse:

$\frac{I_{s p}^{2}}{\left(2 \dot{q}_{F F} / \rho D\right) \eta_{\text {layer }} \eta_{\text {capture }} \eta_{N}\left(D^{2} / \alpha\right)}=\frac{1}{\mathrm{Pe}+\mathrm{Nu}}$

From this balance equations, a proper scale for the specific impulse $I_{s p}$ emerges:

$I_{\text {SpSCALE }}=\sqrt{\frac{2 \dot{q}_{F F}}{\rho D} \eta_{\text {layer }} \eta_{\text {capture }} \eta_{N} \frac{D^{2}}{\alpha}}$

and Eq. (27) can be rewritten as a nondimensional reduced specific impulse:

$I_{s p R E D}=\frac{I_{s p}}{I_{s p S C A L E}}=\sqrt{\frac{1}{\mathrm{Pe}+\mathrm{Nu}}}$

Eqs. (25) and (29) represent the main reference parameters of the engine and can be computed in closed form analytically with the present analysis. In the next section this analytical model will be compared with fully 3D numerical solutions obtained by coupling 


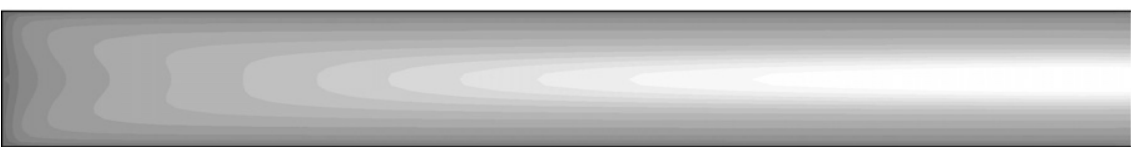

Fig. 6. Temperature distribution $[\mathrm{K}]$ in a module from a 3D numerical computation; walls are kept at 2200 [K].

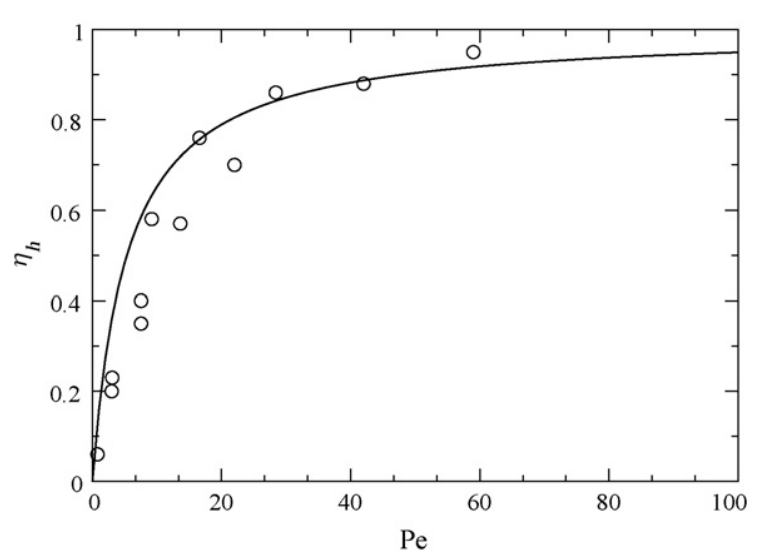

Fig. 7. Comparison of the analytical and numerical heating efficiency. Circles represent numerical 3D results, while the continuos line is the analytical Eq. (25).

CFD codes with a Monte Carlo code for fission fragment energy release with an equation of state for chemical equilibrium.

\subsection{Analytical vs numerical results}

In Fig. 7 the function (continuos line) obtained for the analytical heating efficiency of the rocket module is compared with fully 3D numerical results (circles). The agreement is good with an RMS error of about $12 \%$. The analytical expression can be considered a limiting upper value for the efficiency. This result is justified by the fact that the gas density is higher close to the wall where temperatures are lower. The fission fragments mean free path is shorter where density is higher, and the resulting power per unit volume released by the fission fragments is higher close to the wall. This situation leads to an underestimation of the conductive losses by the analytical model presented in Section 3.2, where a constant density for the gas, and thus a constant power deposition per unit volume, was assumed through the domain. In any case, numerical data collapse with the Peclet number regardless of the tube diam-

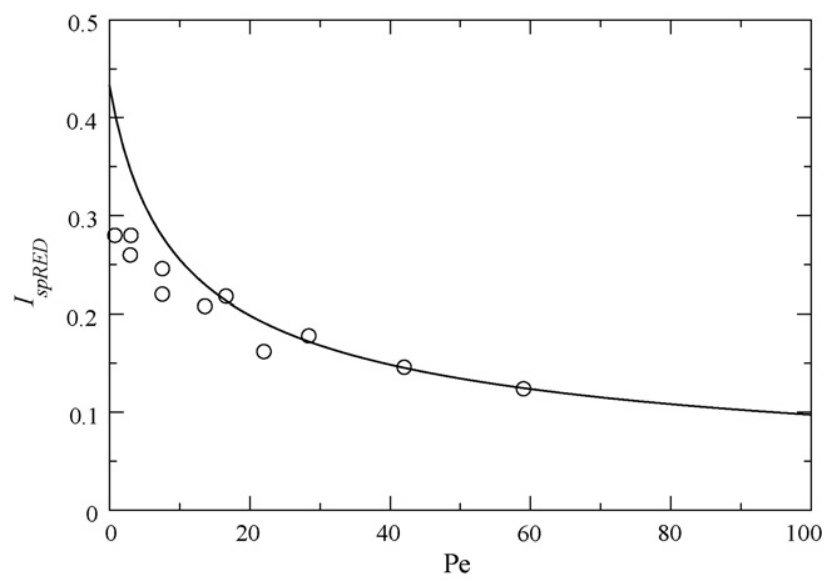

Fig. 8. Comparison of the analytical and numerical reduced specific impulse. Circles represent numerical 3D results, while the continuos line is the analytical Eq. (29). eter; in fact three different values of tube diameters were used in the numerical study (Di Piazza and Mulas, 2006) as well as in other studies documented in Di Piazza and Mulas (2002), Di Piazza et al. (2003), namely $D=0.4,0.6,1$.

Fig. 8 shows a comparison between the analytical solution for the scaled specific impulse (continuos line), and the numerical results (circles) at different Peclet numbers. The agreement is good even for this important performance parameter with an RMS error of about $5 \%$. Therefore, the specific impulse scale $I_{S p S C A L E}$ Eq. (28) and the reported analytical function Eq. (29) can be applied to estimate $I_{s p}$ on the basis of mass flow rate and module diameter.

\section{Conclusions}

An analytical model is presented for the fluid dynamic performance of a single module of a fission fragment rocket engine for interplanetary missions. The analysis shows the crucial role of the Peclet number in rocket engine efficiency and performance. The analytical treatment was based on the integral continuity and energy equations, where all terms were considered and evaluated properly. From these integral equations analytical solutions have been found both for the heating efficiency and for the scaled specific impulse of the engine. These expression are: $\eta_{h}=\mathrm{Pe} /(\mathrm{Pe}+\mathrm{Nu})$, and $I_{s p R E D}=I_{s p} / I_{s p S C A L E}=(\mathrm{Pe}+\mathrm{Nu})^{-0.5}$, where the Nusselt number is a constant given that the flow is fully laminar inside the modules. The Nusselt number is estimated on the basis of the conductive heat transfer, and a value of $16 / 3$ is obtained analytically. The agreement between numerical fully $3 \mathrm{D}$ results and the analytical function is good for both quantities, with RMS errors of $12 \%$ for the efficiency and $5 \%$ for the specific impulse.

\section{References}

Benetti, P., Cinotti, L., Mulas, M., Stalio, R., 2005. Fission fragments direct heating of gas propellant for space rocket. In: DeLuca, L.T., Sackheim, R.L., Palaszewski, B.A. (Eds.), In-Space Propulsion, IWCP, vol. 10. Grafiche GSS, Bergamo, Italy.

Borowski, S.K., Gabris, E.A., Martinelli, J., June 1989. Aerospace America, pp. 16-29.

Chapline, G., 1988. Fission fragment rocket concept. Nucl. Instrum. Methods 271, 207-208.

De Luca, T.L. (Ed.), 2003. Proceedings of the Tenth International Workshop on Combustion and Propulsion: In-Space Propulsion. Lerici, Italy, 21-25 September.

Di Piazza, I., Mulas, M., 2006. Preliminary performance assessment of an unconventional NTR for human missions to mars. AIAA J. Propulsion Power 22 (3), 645-652.

Di Piazza, I., Mulas, M., 2002. Qualitative Analysis of the Performance Parameters of the P242 Fission Fragments Rocket Engine, CRS4 Report, CRS4TECHREP02/44.

Di Piazza, I., Mulas, M., Varone, A., June 2003. Thermo-fluid dynamic analysis of the fission fragments rocket engine. In: Proceedings of the UIT Conference, Trieste, Italy.

Frisbee, R.H., 2003. Advanced space propulsion for the 21st century. J. Propulsion Power 19 (6), 1129-1154.

Grey, J., 2004. Nuclear propulsion and power for space: A roundtable discussion. Aerospace America 17 (11), 26-29.

Howe, S.D., August 2000. Gateway to the Solar System: The Gas Core Nuclear Rocket. Aerospace America.

Kammash, T., Galbraith, D.L., Jan, T.R., 1993. An Americium-fueled gas core nuclear reactor. AIP Conf. 271 (1), 585-589.

Mulas, M., Chibbaro, S., Delussu, G., Di Piazza, I., Talice, M., 2002. Efficient parallel computations of flows of arbitrary fluid for all regimes of Reynolds Mach and Grashof numbers. Int. J. Num. Meth. Heat Fluid Flow 12 (6), 637-657.

Ronen, Y., Shwageraus, E., 2000. Ultra thin ${ }^{242 \mathrm{~m}}$ Am fuel elements in nuclear reactors. Nucl. Instrum. Methods A 455, 442-451.

Rubbia, C., 2000. Fission fragments heating for space propulsion. CERN SL-Note 2000-036 EET. 